\title{
Daily oscillations of skin temperature in military personnel using thermography
}

\author{
Carlos Magno Amaral Costa, ${ }^{1,2}$ M Sillero-Quintana, ${ }^{3}$ S Piñonosa Cano, ${ }^{3}$ \\ D G Moreira, ${ }^{1}$ C J Brito, ${ }^{1}$ A A Fernandes, ${ }^{1}$ G A Pussieldi, ${ }^{1}$ J C B Marins ${ }^{1}$
}

${ }^{1}$ Federal University of Viçosa, Laboratory Human Performance, Minas Gerais, Brazil

${ }^{2}$ Aeronatutics Instruction and Adaptation Center, Minas Gerais, Brazil

${ }^{3}$ Faculty of Sport Sciences, Technical University of Madrid (UPM), Madrid, Spain

\section{Correspondence to} Carlos Magno Amaral Costa, Centro de Instrução e Adaptação da Aeronáutica, Seção de Educação Física, $1^{\circ}$ Ten Carlos Magno Amaral Costa, Belo Horizonte, Minas Gerais CEP 36570-000, Brasil; carlosmagnoac@ig.com.br

Received 29 April 2015 Revised 1 August 2015 Accepted 29 August 2015 Published Online First 18 September 2015

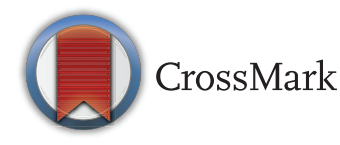

To cite: Costa CMA, SilleroQuintana M, Piñonosa Cano S, et al. J R Army Med Corps 2016;162:335-342.

\begin{abstract}
Introduction The human body makes many physiological adjustments throughout the day, including adjustments to body temperature. The purpose of this study was to determine oscillations in the skin temperature (Tsk-1-Tsk-25) at 25 body regions of interest (ROls) over 1 day using infrared thermography.

Methods Tsk values of 31 male (age 22.9 \pm 3.0 years) Brazilian Air Force members were evaluated from five thermograms collected at 7, 11, 15, 19 and $23 \mathrm{~h}$ $\left(T_{s k}, 11,15,19,23\right)$ by a Fluke imager. We applied one-way analysis of variance for repeated measures for the different times of the day and Tukey's post hoc test to determine significant Tsk differences between ROls $(\alpha=0.05)$, and the cosinor analysis was used to determine the midline estimating statistic of rhythm, amplitude and acrophase of Tsk during the $24 \mathrm{~h}$ period.

Results The anterior hands showed the greatest Tsk variations throughout the day. In the lower limbs, scapula, abdomen, chest and lower back, Tsk-11, Tsk-15, Tsk-19 and Tsk-23 were significantly different $(p<0.05)$ from Tsk-7. The lowest Tsk values were obtained in the early morning, with increases in the afternoon and levelling after 15:00.

Conclusions The Tsk at all ROls and the averaged Tsk showed oscillations throughout the day, with the lowest values in the early morning (07:00). Temperature fluctuations depended on the specific ROI, with thermal stabilisation in some regions in the afternoon and a central upward trend throughout the day in the hands.
\end{abstract}

\section{INTRODUCTION}

The human body undergoes a series of physiological adjustments over a $24 \mathrm{~h}$ cycle. These adjustments are mainly controlled by neurons in the suprachiasmatic nucleus $(\mathrm{SCN}),{ }^{1}$ which is located in the anterior hypothalamus and is responsible for the functional oscillations observed in organs and tissues, such as heart, lung, liver, gut, adrenal gland and adipose tissue. ${ }^{2}$ Thus, the SCN influences rhythmic phenomena that include not only the sleep-wake cycle but also breathing, HR, BP, peristalsis, the motor system, mental functions, nerve activity $^{3}$ and body temperature. ${ }^{4}$

For a correct interpretation of body temperature, it is important to consider its normal fluctuations throughout the day. ${ }^{4}$ This daily variation in body temperature has been demonstrated by measurement of rectal,,$^{5-7}$ axillary, ${ }^{8}$ gastrointestinal, ${ }^{8}$ oral ${ }^{9}$ and skin temperatures (Tsk). ${ }^{10}$ In individuals with normal lifestyles, rectal temperature presents the highest values between 14:00 and 20:00, with a peak at approximately $17: 00$ and a minimum at

\section{Key messages}

- By using infrared thermography (IRT) to perform skin thermographic control, it is possible to map with thermal characteristics considered normal for each body region.

- All analysed regions of interest (ROIs) in our study showed skin temperature (Tsk) oscillations during the day that exhibited different amplitudes.

- The use of IRT for Tsk measurement can aid in the study of heat production and dissipation, which is a key factor in investigations on clothing in the military sector.

- The practical application of IRT in the past few years means it may be a technique capable of preventing, diagnosing and monitoring the process of injuries treatment.

05:00. Similar oscillations at different times of the day are also found with regard to oral temperature. $^{9}$ Small variations of Tsk in the chest and arm region and large swings in the region of the hands were described by Aschoff; ${ }^{4}$ at the distal regions the highest values occurred during the night, when the decline of rectal temperature starts, ${ }^{4}$ these largest differences being present at the extremities because they are areas that have a vasomotor function of heat loss. ${ }^{11}$ Another interesting study on the effect of circadian rhythm described different values of Tsk depending on the body area examined; the proximal area followed the same circadian rhythm rectal temperature, while the Tsk distal, hands and feet, exhibited an opposite pattern. ${ }^{12}$

One of the problems encountered by Tsk studies employing thermal sensors is the small number of body regions analysed, ${ }^{10}{ }^{13}$ which makes it difficult to understand what occurs throughout the whole body. Therefore, it is necessary to use assessment tools that allow simultaneous monitoring of Tsk in a wide range of body regions.

Infrared thermography (IRT) is a safe, noninvasive and low-cost technique that allows for the rapid and non-contact recording of the irradiated energy released from the body. ${ }^{14}{ }^{15} \mathrm{~A}$ highresolution thermal image can provide interesting information about the complex thermoregulation system of the body. The development of fast and easy-to-use IRT monitoring tools allows researchers to obtain the general or local thermal profiles of human bodies. 
It is worth noting that despite the findings of van den Heuvel et $a l,{ }^{16}$ which showed a positive correlation between the temperatures obtained by IRT and contact thermal sensors, these two methods exhibited significant differences in Tsk measurements in another study, with the IRT values that were $1.3-3.4^{\circ} \mathrm{C}$ lower than those of contact thermal sensors. ${ }^{16}$

The differences between these methods are caused by differences in the protocols. On the one hand, thermal sensors make physical contact with the skin, capturing the heat released from the body through conduction processes, whereas IRT records the temperature distribution on the skin via a thermal imager that captures and processes the long-frequency infrared radiation emitted by the body surface. ${ }^{17}$

Although there is extensive evidence of variations in body temperature as measured by different techniques, a MEDLINE database search for the keywords 'circadian rhythm and thermography' or 'periods of the day and thermography' revealed no studies that used IRT to measure Tsk throughout the day.

The use of IRT for Tsk measurement can aid in the study of heat production and dissipation, ${ }^{18}$ which are key factors in investigations on clothing in both the military sector and the field of competitive or recreational exercise; such clothing aims to facilitate heat loss or retention depending on the intensity of the exercise and the weather conditions. Because of previous studies that demonstrate daily variations of Tsk in different body regions and their heat loss functions, it is important to identify simultaneously these variations throughout the body. Therefore, we selected 25 regions of interest (ROIs) that correspond to the right/left sides and anterior/posterior regions of the body, areas normally covered by sports or military clothing.

Therefore, the purpose of our study is to record the Tsk oscillations over a period of 1 day in young men in the military by IRT in 25 ROIs covering the whole body.

\section{METHODS}

\section{Subjects}

A convenience sample of 31 males enlisted in the Brazilian Air Force School of Aeronautics, São Paulo, Brazil, volunteered for this study. All men were considered active as they performed military physical training sessions of moderate to high intensity 4-5 times per week.

The exclusion criteria were (a) any bone, muscle or joint injury in the two months prior to data collection; (b) history of kidney problems due to fluid accumulation in the body, causing inflammation in several body regions; (c) currently undergoing physiotherapy treatment; (d) tobacco or drug consumption (antipyretics or diuretics), or food supplements that might interfere with homeostasis hydration or body temperature in the previous two weeks; (e) burns on the skin, regardless of their degree; (f) undergoing any local treatment with creams, ointments or lotions; and (g) pain, fever symptoms, or sleeping disturbances in the previous seven days.

The assessed men participated voluntarily and remained on the military base during the $32 \mathrm{~h}$ study period. After being informed about the dynamics of the study and its objectives, they signed consent forms to participate and did not receive any financial compensation.

\section{Procedure and study design}

The data were collected in two spring season days at an external average temperature of $25^{\circ} \mathrm{C}$. The standardisation of the evaluation conditions began the day before data collection and all subjects performed no high-intensity physical exercises ${ }^{19}$ and were restricted to only normal daily activities. After the evening meal, which was held at the military base, the subjects retired into their respective rooms between 21:30 and 22:00 for a period of $8 \mathrm{~h}$ of sleep.

Thermographic images were collected the next day between 07:00 and 24:00 at five different times with $3 \mathrm{~h}$ intervals between collections $(07: 00$ to $08: 00,11: 00$ to $12: 00,15: 00$ to $16: 00 ; 19: 00$ to $20: 00$ and 23:00 to 00:00). During breaks, the subjects remained in their rooms and engaged in only sedentary activities with low-energy consumption, such as watching TV, reading or playing cards $(<1.6 \mathrm{METs}) .{ }^{19}$

Subjects ate four meals, always soon after the completion of thermographic imaging and $2 \mathrm{~h}$ before the next collection of images. This schedule was maintained to minimise any thermogenic effect caused by food. Liquid consumption was restricted to water. Figure 1 summarises the study protocol.

The Tsk values of the ROIs were obtained from the thermograms according to the criteria described by the European Association of Thermology: ${ }^{20}$ the first step consisted of preparing a room on the military base $(4 \times 6 \times 2.6 \mathrm{~m})$ with no natural light, a temperature $23^{\circ} \mathrm{C} \pm 1{ }^{\circ} \mathrm{C}$ and humidity of $50 \% \pm 5 \%$. These laboratory conditions were similar to other studies. ${ }^{21-23}$ All the thermograms were performed by the same examiner using the same imager, which was positioned on a tripod $4 \mathrm{~m}$ from the subject.

For each of the five data collections, the subjects arrived and were instructed to change into a swimsuit or shorts. After a minimum of $10 \mathrm{~min}$ of adaptation to the room conditions, as recommended by Marins et $a l,{ }^{21}$ the thermograms were recorded.

During the adaptation period and the data collection, the subjects were asked to abstain from making any type of movement, sitting, crossing legs or arms, or scratching, given that those actions can modify the local Tsk by friction. Before the first thermography recording, the standard questionnaire used by Marins $e t a l^{21}$ was completed to exclude subjects meeting any exclusion criteria. After these preparatory steps, thermograms were taken following the procedures described below. The subject was positioned standing in anatomical position (Figure 2) facing the imager. Four images were recorded: anterior and posterior views of the lower limbs and upper limbs.

The ROIs analysed included the left and right hand, forearm, upper arm, thigh and leg. In addition, Tsk of the chest, abdomen, lower back and upper back were collected. The choice of ROIs was based on an earlier work by Marins et al. ${ }^{21}$ Tsk values averaged from the 25 ROIs were collected by the software Smartview (Fluke, Everett, USA). The shape of each
Figure 1 Schedule of data collection. RES, rest in their rooms; IRT- $n=$ data collection number; IRT, infrared thermography; EAT-1=breakfast; EAT-2=lunch; EAT-3=snack; EAT-4=dinner.

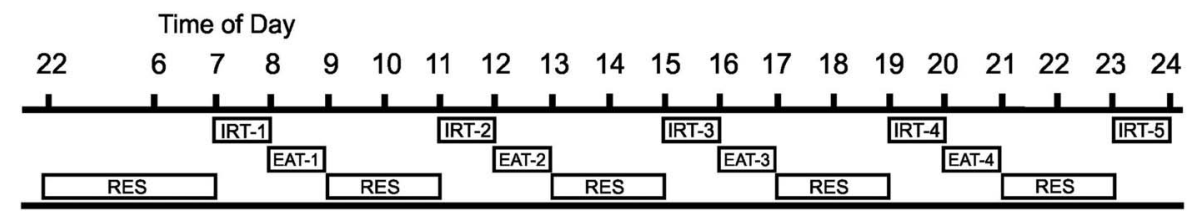



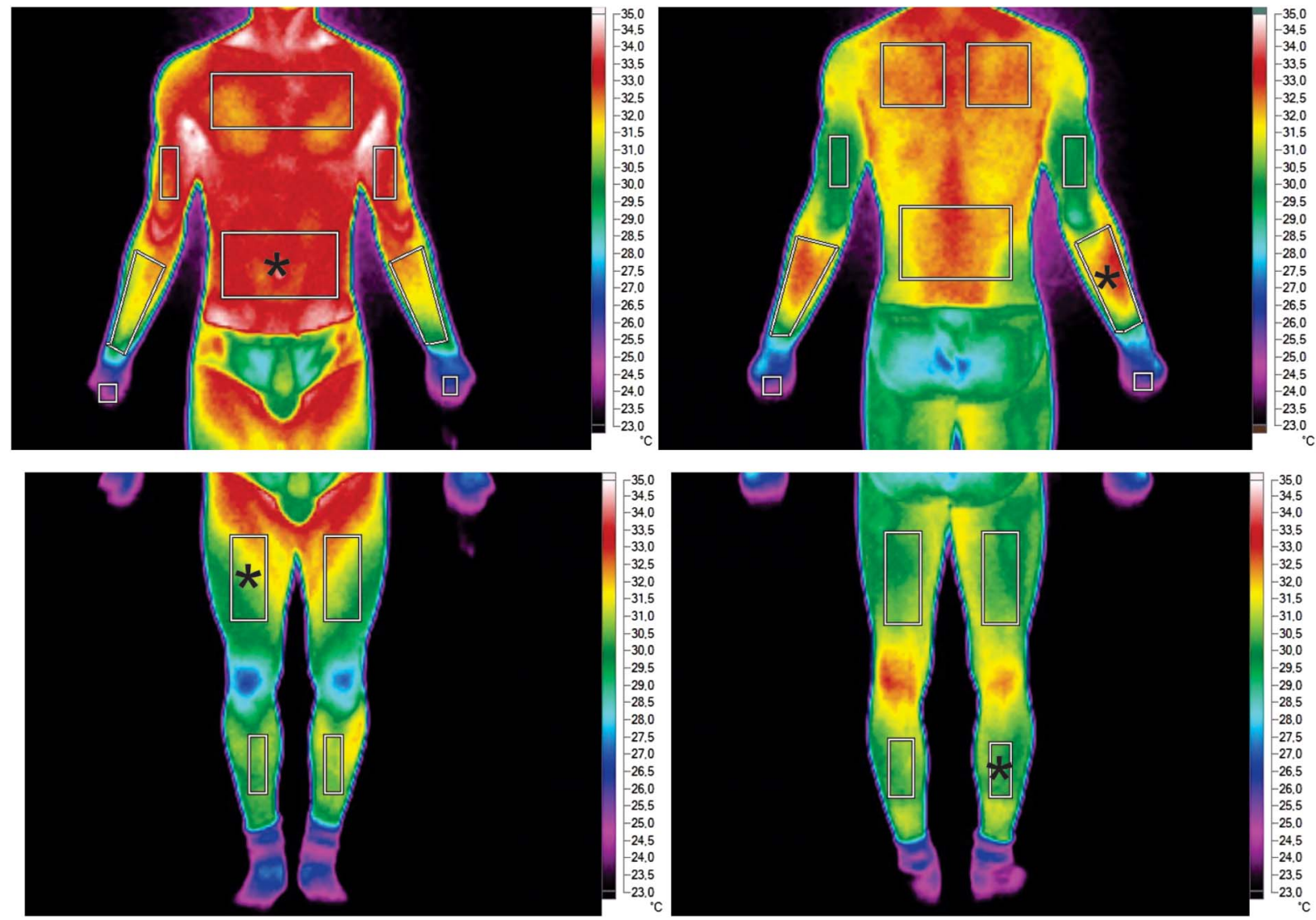

Figure 2 Front and back infrared thermograms and the locations of the rectangles demarcating the 25 regions of interest (ROIs) analysed.

*indicates the four ROIs used to calculate the average skin temperature.

ROI rectangle was determined by anatomical landmarks (Table 1).

Figure 2 shows an example of how the images were acquired and markings made of 25 ROIs.

The average Tsk (AvTsk) was calculated using the formula AvTsk $=0.34 \times \mathrm{Tsk}_{\mathrm{abd}}+0.15 \times \mathrm{Tsk}_{\mathrm{f}-\mathrm{arm}}+0.33 \times \mathrm{Tsk}_{\text {thigh }}+0.18 \times \mathrm{Tsk}_{\text {leg }}$ proposed by Choi et al $^{24}$ using temperature values for only 4 of the 25 ROIs analysed in our study; these regions are the abdomen $\left(T s k_{\mathrm{abd}}\right)$, posterior forearm right $\left(\mathrm{Tsk}_{\mathrm{f}-\mathrm{arm}}\right)$, anterior thigh right $\left(\mathrm{Tsk}_{\text {thigh }}\right)$ and posterior leg right $\left(\mathrm{Tsk}_{\mathrm{leg}}\right)$ (Figure 2$)$.

\section{Equipment}

The thermographic imager was an IRT-25 camera (Fluke, Everett, USA) with a measurement range of $-20^{\circ} \mathrm{C}$ to $+350^{\circ} \mathrm{C}$, an accuracy of $\pm 2^{\circ} \mathrm{C}$ or $2 \%$ of the measurement, a sensitivity of

Table 1 Definition of the anatomical landmarks used

\begin{tabular}{|c|c|}
\hline & Landmarks \\
\hline Hand & $\begin{array}{l}\text { The junction of the third metacarpal proximal } \\
\text { phalanx with the third ulnar styloid process }\end{array}$ \\
\hline Forearm & Cubital fossa and distal forearm \\
\hline Arm & Cubital fossa and axillary line \\
\hline Abdomen (and low back) & Xiphoid process and $5 \mathrm{~cm}$ below the umbilicus \\
\hline Chest (upper back) & Nipple line and top edge of the sternum \\
\hline Thigh & $\begin{array}{l}5 \mathrm{~cm} \text { above the upper border of the patella and the } \\
\text { inguinal line }\end{array}$ \\
\hline Leg & $\begin{array}{l}5 \mathrm{~cm} \text { below the lower border of the patella and } \\
10 \mathrm{~cm} \text { above the malleolus }\end{array}$ \\
\hline
\end{tabular}

$<0.1^{\circ} \mathrm{C}$, infrared spectral bands from 7.5 to $14 \mu \mathrm{m}$, a refresh rate of $9 \mathrm{~Hz}$ and a resolution of $160 \times 120$ pixels (focal plane array). The images were obtained at the interval the minimum cycle of calibration of 2 years performed by the manufacturer, being considering a skin emissivity of $0.98^{17}$ and analysed using software Smartview, V.3.1. The thermographic imager requires a short time of approximately $5 \mathrm{~s}$ of stabilisation after being connected; the focus was adjusted according to the distance from the subject. The temperature and humidity of the room were recorded by a thermohygrometer (ITHT-2200; ranges: temperature $=10-50^{\circ} \mathrm{C} \pm 1{ }^{\circ} \mathrm{C}$, humidity $\left.=20-90 \% \pm 5 \%\right)$. An air conditioner (Consul $10000 \mathrm{BTU}$ Hot/Cold CCO10B) was used to maintain the environmental conditions of the room.

\section{Statistical analyses}

After confirming the normal distribution of the variables (Shapiro-Wilk test) and homogeneity of variances (F-test), descriptive statistics, including mean and SD, were used to present the data. Additionally, one-way analysis of variance for repeated measures, followed by Tukey's post hoc test, was performed to determine significant differences between the Tsk for each ROI at different times of day. To validate the repeated measures, we adopted the Mauchly sphericity test, and where necessary, the Greenhouse-Geisser correction was used. The cosinor analysis was used to determine the midline estimating statistic of rhythm (MESOR), amplitude and acrophase of Tsk during the $24 \mathrm{~h}$ period. The MESOR is the average value of the fitted cosine curve. A significance level of $\alpha=0.05$ was established. The software Cosinor Periodrogam 2.7 was used for cosinor analysis, and the software SigmaPlot 11.0 was used for all other calculations. 


\section{RESULTS}

The mean age of the study participants: $22.9 \pm 3$ years; height: $178.3 \pm 7.8 \mathrm{~cm}$; body mass: $73.4 \pm 8.2 \mathrm{~kg}$; body composition $9.6+3.5 \%$. Figures 3 and 4 show the mean and SD of the Tsk values for the analysed ROIs at different times of the day. Each ROI exhibited significant Tsk differences $(p<0.05)$ between at least two time points. Additionally, each ROI showed a specific Tsk behaviour, such that the average values were lower in the distal hands, forearms, arms, legs and thigh regions compared with the chest, abdomen and dorsal scapular inferior.

The Tsk of the anterior and posterior forearm and arm, abdomen and lower back showed no significant differences between 07:00 and 11:00 or between 15:00, 19:00 and 23:00, with the exception of the anterior forearm, which showed a significant difference $(\mathrm{p}<0.05)$ between 19:00 and 23:00.

In the posterior and anterior thigh and legs, chest and scapula, the Tsk at 11:00, 15:00, 19:00 and 23:00 were significantly different $(p<0.05)$ from those at 07:00, demonstrating an increase in Tsk in the morning and a stabilisation after 11:00. The Tsk of the anterior thigh was also significantly different $(p<0.05)$ at $11: 00$ compared with $15: 00$, as was the anterior leg at 11:00 compared with 19:00.

Table 2 shows the AvTsk obtained using the Tsk values using temperature from values only 4 of the 25 ROIs analysed in our study; these regions are abdomen (Tsk-12), posterior forearm right (Tsk-15), anterior thigh right (Tsk-7) and posterior leg right (Tsk-21) at the five time points. The results show that AvTsk also varied during the period analysed. The lowest values were obtained in the early morning, with increases in the afternoon and a levelling off starting at 15:00. AvTsk was significantly different $(p<0.05)$ at 07:00 and 11:00 compared with 15:00, 19:00 and 23:00; however, the latter time points were not significantly different from each other. AvTsk was also significantly different $(p<0.05)$ between 07:00 and 11:00.
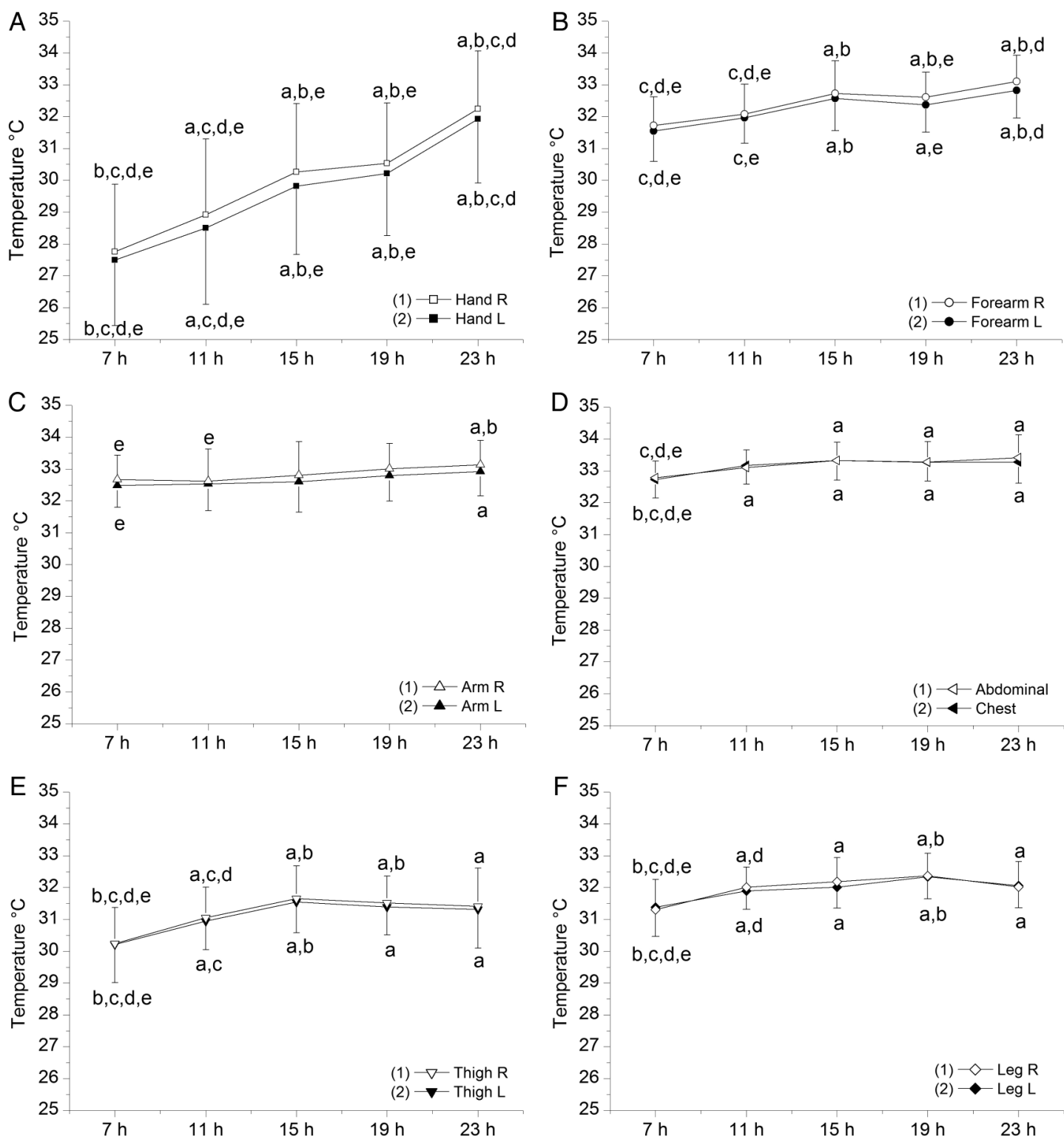

Figure 3 Skin temperature values at anterior regions of interest (ROIs) in men $(n=31)$. Significant differences between individual time points are represented by the letters a-e. a, from 07:00; b, from 11:00; c, from 15:00; d, from 19:00; e, from 23:00. The top letters of each graph correspond to ROI number 1 . The lower letters of each graph correspond to ROI number 2 . 

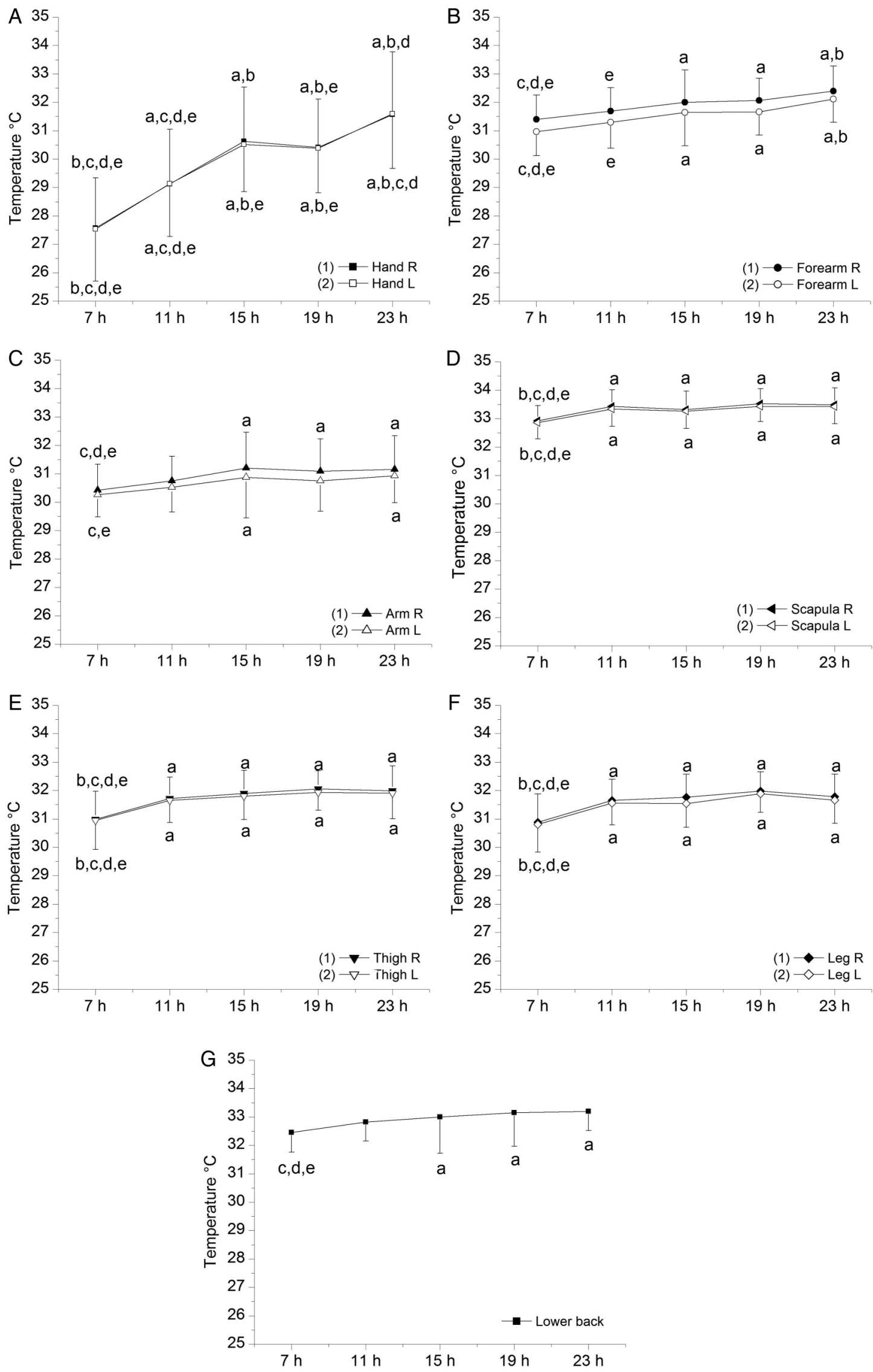

Figure 4 Skin temperature values at posterior regions of interest (ROIs) in men $(n=31)$. Significant differences between individual time points are represented by the letters a-e. a, from 07:00; b, from 11:00; c, from 15:00; $d$, from 19:00; e, from 23:00. The top letters of each graph correspond to ROI number 1 . The lower letters of each graph correspond to ROI number 2. 
Table 2 Average skin temperature (AvTsk) calculated from the Tsk of four regions of interest using the Choi et al formula ${ }^{24}$

\begin{tabular}{lccccc}
\hline Hour of the day & $\mathbf{0 7 : 0 0}(\mathrm{a})$ & $\mathbf{1 1 : 0 0}(\mathrm{b})$ & $\mathbf{1 5 : 0 0}(\mathrm{c})$ & $\mathbf{1 9 : 0 0}(\mathrm{d})$ & $\mathbf{2 3 : 0 0}(\mathrm{e})$ \\
\hline AvTsk $\left({ }^{\circ} \mathrm{C}\right)$ & $31.38^{*}$ & $31.92 \dagger$ & $32.26^{*} \dagger$ & $32.24^{*} \dagger$ & $32.28^{*} \dagger$ \\
SD & 0.73 & 0.57 & 0.74 & 0.63 & 0.83 \\
Maximum $\left({ }^{\circ} \mathrm{C}\right)$ & 29.44 & 30.82 & 30.79 & 31.01 & 30.29 \\
Minimum $\left({ }^{\circ} \mathrm{C}\right)$ & 32.78 & 32.78 & 33.50 & 33.40 & 33.97 \\
\hline
\end{tabular}

*Significant difference $(p<0.05)$ from the value of 11:00. Formula:

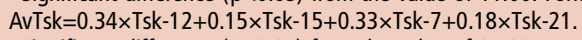
tSignificant difference $(p<0.05)$ from the value of 07:00.

Table 3 shows the rhythmic parameters of the cosinor analysis The acrophase denotes the time after midnight when the fitted curve is estimated to be at its peak. The amplitude is the distance between the maximum of the fitted curve and the MESOR. ${ }^{25}$ The MESORs indicate a similarity in Tsk values between the left and right sides of the body; the largest amplitudes are in the hands; and the acrophases show that ROIs have highest Tsk values in the late afternoon and evening, even though they differ in detail with ROI. The anterior parts of the right and left arms showed significant rhythms $(\mathrm{p}<0.05)$.

\section{DISCUSSION}

The temperature values obtained by IRT revealed Tsk variations in all examined ROIs over the five analysed times of day; these variations exhibited different magnitudes depending on the area analysed. Our findings are similar to those of studies that identified daily variations in body temperature measured from the rectum, ${ }^{5-7}$ axilla $^{68}$ gastrointestinal region, ${ }^{8}$ mouth ${ }^{9}$ and skin. ${ }^{13}$
It seems clear that body temperature varies throughout the day and exhibits a wave-shaped progression, which has been confirmed in the studies cited above that analysed a limited number of ROIs. Our study (Figures 3 and 4) shows that such changes also occur in the whole body, although their magnitudes are different at the different ROIs, especially in the extremities relative to the trunk. This observation justifies considering the temperature behaviour of each body region individually, demonstrating acrophases between late afternoon and evening (Table 3).

The Tsk values obtained in this study were consistently lower in the morning (07:00): in 23/25 analysed ROIs, the lowest Tsk was obtained at this time (Figures 3 and 4), whereas 18 ROIs showed the highest temperatures at 23:00. A lower body temperature in the morning, followed by an increase in the afternoon, has also been observed by other authors using different methods for recording body temperature. Some examples are the works of Monk et al, ${ }^{5}$ who registered rectal temperature in young adults and elderly individuals; Edwards et al, ${ }^{9}$ who used a sublingual clinical thermometer; and Pronina and Ribakov, ${ }^{13}$ who measured the Tsk through a sensor attached to the shoulder of children and young adults.

Although the use of thermocouples has advantages such as high precision, sensitivity, reproducibility, high response time and high amplitude temperature measurement, ${ }^{26} 27$ it also has disadvantages, such as reading the Tsk over only a restricted area of a few square centimetres, ${ }^{26}$ the relatively high cost of some basic reading equipment, ${ }^{26}$ the differences of Tsk generated by using fixing thermocouples ${ }^{28}$ and difficulties due to heat loss through convection and evaporation in the region where

Table 3 Analysis cosinor in the 25 analysed regions of interest (ROls) (Tsk-1-Tsk-25)

\begin{tabular}{|c|c|c|c|c|c|}
\hline ROI & Anterior ROI & MESOR $\left({ }^{\circ} \mathrm{C}\right)$ & Amplitude $\left({ }^{\circ} \mathrm{C}\right)$ & Acrophase & p Value \\
\hline Tsk-1 & Right hand & 29.6 & 1.75 & $21: 48$ & 0.234557 \\
\hline Tsk-2 & Left hand & 29.9 & 1.75 & $21: 28$ & 0.261442 \\
\hline Tsk-3 & Right forearm & 32.3 & 0.49 & $20: 16$ & 0.299920 \\
\hline Tsk-4 & Left forearm & 32.4 & 0.56 & $20: 52$ & 0.279506 \\
\hline Tsk-5 & Right arm* & 32.7 & 0.21 & $22: 44$ & 0.036082 \\
\hline Tsk-6 & Left arm* & 32.4 & 0.56 & $20: 52$ & 0.007917 \\
\hline Tsk-7 & Right thigh & 31.0 & 0.68 & $18: 32$ & 0.108469 \\
\hline Tsk-8 & Left thigh & 31.1 & 0.71 & $18: 20$ & 0.135619 \\
\hline Tsk-9 & Right leg & 31.9 & 0.38 & $19: 24$ & 0.149677 \\
\hline Tsk-10 & Left leg & 31.9 & 0.52 & $18: 12$ & 0.089852 \\
\hline Tsk-11 & Chest & 33.1 & 0.29 & $18: 36$ & 0.261255 \\
\hline Tsk-12 & Abdomen & 33.2 & 0.26 & $19: 52$ & 0.224596 \\
\hline ROI & Posterior ROI & MESOR $\left({ }^{\circ} \mathrm{C}\right)$ & Amplitude $\left({ }^{\circ} \mathrm{C}\right)$ & Acrophase & p Value \\
\hline Tsk-13 & Right hand & 29.8 & 1.59 & $20: 28$ & 0.273843 \\
\hline Tsk-14 & Left hand & 29.7 & 1.62 & $20: 32$ & 0.273904 \\
\hline Tsk-15 & Right forearm & 31.9 & 0.40 & $21: 16$ & 0.216061 \\
\hline Tsk-16 & Left forearm & 31.5 & 0.43 & $21: 36$ & 0.247159 \\
\hline Tsk-17 & Right arm & 31.0 & 0.38 & $19: 20$ & 0.192136 \\
\hline Tsk-18 & Left arm & 30.6 & 0.26 & $19: 40$ & 0.331987 \\
\hline Tsk-19 & Right thigh & 33.4 & 0.19 & $11: 16$ & 0.308451 \\
\hline Tsk-20 & Left thigh & 33.2 & 0.21 & $19: 12$ & 0.328707 \\
\hline Tsk-21 & Right leg & 31.8 & 0.50 & $19: 12$ & 0.170138 \\
\hline Tsk-22 & Left leg & 31.6 & 0.41 & $19: 00$ & 0.270348 \\
\hline Tsk-23 & Right scapula & 31.6 & 0.49 & $18: 36$ & 0.198073 \\
\hline Tsk-24 & Left scapula & 31.5 & 0.43 & $19: 08$ & 0.318177 \\
\hline Tsk-25 & Lower back & 32.9 & 0.34 & $20: 08$ & 0.203623 \\
\hline
\end{tabular}


the thermocouple is fixed. ${ }^{28} 29$ In addition, long-term activities, sweating and body movement can cause the thermocouple detachment, ${ }^{29}$ interference due to movement of the attachment cable ${ }^{26}$ and difficulty of use in a military context due to interference from electronic devices. The use of the IRT can serve as an alternative method to minimise the disadvantages of thermocouples because it is a non-invasive technique that does not require physical contact..$^{23}{ }^{30}$ The monitoring of Tsk can be focused on a particular region of the body for local analysis or can assess the entire body, enabling a more comprehensive analysis. ${ }^{30} 23$ Other advantages include freedom of movement during exercise and lack of interference of processes concerned with heat loss through radiation, convection and evaporation. The method measures heat loss as it occurs naturally, has high sensitivity, accuracy and reproducibility, and can be recorded by a camera. ${ }^{23} 3031$ However, disadvantages are the need for care regarding factors that can interfere with the collection of IRT data (such as room temperature and light radiation), the need to train individuals in the use of the software that analyses the IRT and circumstances when measurements require activities being performed at any given time to be stopped in order to perform the IRT. ${ }^{23}$

Considering the agreement of our study, we think that IRT might be an alternative tool to assess the temperature variations of Tsk when several body areas need to be simultaneously measured.

Several metabolic adjustments may explain the lower temperature in the early morning compared with other times of the day. According to Wakamura and Tokura, ${ }^{32}$ the sharp reduction in metabolic rate that occurs while the subject sleeps at night generates a decrease in body temperature. Dijk et $a l^{7}$ reported that endocrine responses of melatonin and cortisol are also involved in this process. The administration of melatonin normally leads to a reduction in the internal temperature under resting conditions, ${ }^{33}$ suggesting that the nocturnal secretion of melatonin may play a role in the diurnal variations of body temperature. ${ }^{34}$

The major differences recorded throughout the day (Figures 3 and 4) and the larger amplitude (Table 3) occurred in the distal limbs, especially in the hands, which may be explained by the fact that they are the sites where vasomotor function allows heat loss. ${ }^{11}$

Despite such increases in Tsk in the distal regions, the values in these regions did not surpass the Tsk values in regions of the trunk, which remained quite constant throughout the day, with lower amplitude in the chest and abdomen (Table 3). This lower amplitude of Tsk in the central body can be regarded as normal, given the concentration of major organs in the abdomen and chest, which, under resting conditions, are the main producers of heat. ${ }^{35}$ Another study corroborates the results obtained in this work, as it also reported higher Tsk values in the abdominal area in adults and elderly people. ${ }^{36}$

Our results confirm the existence of a thermal plateau between 15:00 and 23:00 in the anterior and posterior arms, thighs and legs, scapulas, lower back, chest and abdomen. The peak temperatures in the Tsk-5 and Tsk-6 (Figure 3C), Tsk-11 and Tsk-12 (Figure 3D), Tsk-9 and Tsk-10 (Figure 3F), Tsk-23 and Tsk-24 (Figure 4D), Tsk-19 and Tsk-20 (Figure 4E), Tsk-21 and Tsk-22 (Figure 4F) occurred between 15:00 and 23:00, demonstrated also by acrophase obtained through cosinor analysis of each ROI. A body temperature peak at approximately 17:00 has been reported by other authors who recorded the Tsk in proximal areas using rectal temperature and oral temperature. ${ }^{9}$

Aschoff ${ }^{4}$ described the small variations of Tsk in the region of the chest and arms and wide oscillations of Tsk in hands, which reached a maximum at 22:00, when rectal temperature started to decline. ${ }^{4}$ These results agree with those obtained in the present study (Figures 3A, B and 4A, B), in which the highest Tsk in the distal regions (ie, Tsk-1, Tsk-2, Tsk-3, Tsk-4, Tsk-13, Tsk-14, Tsk-15 and Tsk-16) occurred between 20:00 and 22:00 (Table 3).

The distribution of Tsk should exhibit symmetry between contralateral hands, forearms, arms, thigh and legs because the thermoregulatory adjustments of the right and left sides should be similar due to a balanced nerve stimulation. The present study noted similar behaviour between each pair of ROIs in the bilateral analysis and demonstrated by the similarity of rhythmic parameters (MESOR, amplitude and acrophase).

The development of new equipment collaborated, coupled with the evolution of the practical application of IRT in the past few years, has become an innovative feature, and it may be a technique capable of preventing, diagnosing and monitoring the process of injury treatment. ${ }^{37} 38$ The lack of similar studies employing IRT throughout the day makes it difficult to generalise our results, but the present study is valuable in establishing the differences and the parameters of the circadian rhythm and the Tsk pace at five times of the day using the IRT technique. Our findings should stimulate further work to investigate the thermographic profile of the skin during the day in different age groups and genders, considering that there are specific thermal responses in children, women and the elderly. ${ }^{13}$ This restricted sample population can be considered a limitation of this study, as can the absence of data between 23:00 and 07:00 and the lack of data about the internal temperature (ie, gastrointestinal or rectal), which would provide more information for a more precise interpretation of body temperature variations.

\section{CONCLUSIONS}

By using IRT to perform skin thermographic control, it is possible to establish the specific thermal profile of subjects generating a map with thermal characteristics considered normal for each body region. It is important to consider that all analysed ROIs in our study showed Tsk oscillations during the day but exhibited different amplitudes. Distal ROIs showed greater Tsk variation than central regions. Lower Tsk values were detected in early morning, while during the night the highest temperatures were recorded at most of the ROIs. The AvTsk also indicated the existence of thermal variations during the day.

Contributors CMAC, JCBM, DGM and AdAF, contributed to the planning, methodology and complement all the work and writing of the article, and are members of Research Lab. MS-Q and SPC, contributed with article writing, translation and proofreading. CJ and GdAP contributed to the review statistical analysis and final work writing

Funding A post-doctoral fellowship of the Conselho Nacional de Pesquisa (CNPq) -Brazil.

Competing interests None declared.

\section{Patient consent Obtained.}

Ethics approval The ethics committee of the Viçosa Federal University, Brazil, approved the study procedures (protocol number: 40928260540), which followed the principles outlined by the World Medical Assembly Declaration of Helsinki.

Provenance and peer review Not commissioned; externally peer reviewed.

\section{REFERENCES}

1 Siepka SM, Yoo SH, Park J, et al. Genetics and neurobiology of circadian clocks in mammals. Cold Spring Harb Symp Quant Biol 2007;72:251-9.

2 Garaulet M, Madrid JA. Chronobiology, genetics and metabolic syndrome. Curr Opin Lipidol 2009;20:127-34 
3 Junker U, Wirz S. Review article: chronobiology: influence of circadian rhythms on the therapy of severe pain. J Oncol Pharm Pract 2010;16:81-7.

4 Aschoff J. Circadian control of body-temperature. J Thermal Biology 1983;8:143-7.

5 Monk TH, Buysse DJ, Reynolds CF, III, et al. Circadian temperature rhythms of older people. Exp Gerontol 1995;30:455-74.

6 Thomas KA, Burr R, Wang SY, et al. Axillary and thoracic skin temperatures poorly comparable to core body temperature circadian rhythm: results from 2 adult populations. Biol Res Nurs 2004;5:187-94.

7 Dijk DJ, Duffy JF, Silva EJ, et al. Amplitude reduction and phase shifts of melatonin, cortisol and other circadian rhythms after a gradual advance of sleep and light exposure in humans. PLOS ONE 2012;7:e30037.

8 Edwards B, Waterhouse J, Reilly $\mathrm{T}$, et al. A comparison of the suitabilities of rectal, gut, and insulated axilla temperatures for measurement of the circadian rhythm of core temperature in field studies. Chronobiol Int 2002;19:579-97.

9 Edwards B, Waterhouse J, Reilly T. Circadian rhythms and their association with body temperature and time awake when performing a simple task with the dominant and non-dominant hand. Chronobiol Int 2008;25:115-32.

10 Le Fur I, Reinberg A, Lopez $\mathrm{S}$, et al. Analysis of circadian and ultradian rhythms of skin surface properties of face and forearm of healthy women. J Invest Dermatol 2001;117:718-24.

11 Machado-Moreira CA, Caldwell JN, Mekjavic IB, et al. Sweat secretion from palmar and dorsal surfaces of the hands during passive and active heating. Aviat Space Environ Med 2008;79:1034-40.

12 Krauchi K, Wirz-Justice A. Circadian rhythm of heat production, heart rate, and skin and core temperature under unmasking conditions in men. Am J Physiol 1994;267 (3 Pt 2):R819-29.

13 Pronina TS, Rybakov BP. Features of the circadian rhythm of temperature of the skin at children of 8-9 years and young men and girls. Fiziol Cheloveka 2011;37:98-104.

14 Fernández-Cuevas I, Marins JCB, Arnáiz Lastras J, et al. Classification of factors influencing the use of infrared thermography in humans: a review. Infrared Phys Technol 2015;71:28-55.

15 Jiang $\mathrm{L}, \mathrm{Ng} \mathrm{EY}$, Yeo AC, et al. A perspective on medical infrared imaging. J Med Eng Technol 2005;29:257-67.

16 van den Heuvel CJ, Ferguson SA, Dawson D, et al. Comparison of digital infrared thermal imaging (DITI) with contact thermometry: pilot data from a sleep research laboratory. Physiol Meas 2003;24:717-25.

17 Jones BF. A reappraisal of the use of infrared thermal image analysis in medicine. IEEE Trans Med Imaging 1998;17:1019-27.

18 Ferreira JJ, Mendonca LC, Nunes LA, et al. Exercise-associated thermographic changes in young and elderly subjects. Ann Biomed Eng 2008;36:1420-7.

19 Norton K, Norton L, Sadgrove D. Position statement on physical activity and exercise intensity terminology. I Sci Med Sport 2010;13:496-502.

20 Ring EF, Kurt A. Standard Procedures for Infrared Imaging in Medicine. Vol 36. Medical Devices and Systems: CRC Press, 2006:1-14.
21 Marins JCB, Moreira DG, Cano SP, et al. Time required to stabilize thermographic images at rest. Infrared Phys Technol 2014;65:30-5.

22 Marins JC, Fernandes AA, Cano SP, et al. Thermal body patterns for healthy Brazilian adults (male and female). J Therm Biol 2014;42:1-8.

23 Fernandes AA, Amorim PR, Brito CJ, et al. Measuring skin temperature before, during and after exercise: a comparison of thermocouples and infrared thermography. Physiol Meas 2014;35:189-203.

24 Choi JK, Miki K, Sagawa S, et al. Evaluation of mean skin temperature formulas by infrared thermography. Int I Biometeorol 1997;41:68-75.

25 Refinetti R, Lissen GC, Halberg F. Procedures for numerical analysis of circadian rhythms. Biol Rhythm Res 2007;38:275-325.

26 Smith AD, Crabtree DR, Bilzon JL, et al. The validity of wireless iButtons and thermistors for human skin temperature measurement. Physiol Meas 2010;31:95-114

27 Hasselberg MJ, McMahon J, Parker K. The validity, reliability, and utility of the iButton(R) for measurement of body temperature circadian rhythms in sleep/wake research. Sleep Med 2013;14:5-11.

28 Tyler CJ. The effect of skin thermistor fixation method on weighted mean skin temperature. Physiol Meas 2011;32:1541-7.

29 Buono MJ, Jechort A, Marques R, et al. Comparison of infrared versus contact thermometry for measuring skin temperature during exercise in the heat. Physiol Meas 2007;28:855-9.

30 Vargas JVC, Brioschi ML, Dias FG, et al. Normalized methodology for medical infrared imaging. Infrared Phys Technol 2009;52:42-7.

31 Bouzida N, Bendada A, Maldague XP. Visualization of body thermoregulation by infrared imaging. J Thermal Biol 2009;34:120-6.

32 Wakamura T, Tokura H. Circadian rhythm of rectal temperature in humans under different ambient temperature cycles. I Thermal Biol 2002;27:439-47.

33 Cagnacci A, Krauchi K, Wirz-Justice A, et al. Homeostatic versus circadian effects of melatonin on core body temperature in humans. J Biol Rhythms 1997;12:509-17.

34 Johnson JM, Kellogg DL Jr. Thermoregulatory and thermal control in the human cutaneous circulation. Front Biosci (Schol Ed) 2010;2:825-53.

35 Campbell I. Body temperature and its regulation. Anaesth Intensive Care Med 2011;12:240-4.

36 Niu HH, Lui PW, Hu JS, et al. Thermal symmetry of skin temperature: normative data of normal subjects in Taiwan. Zhonghua Yi Xue Za Zhi (Taipei) 2001;64:459-68.

37 Hildebrandt C, Raschner C, Ammer K. An overview of recent application of medical infrared thermography in sports medicine in Austria. Sensors 2010;10:4700-15.

38 Marins JCB, Fernandes AdA, Moreira DG, et al. Thermographic profile of soccer players' lower limbs. Rev Andal Med Deport 2014;7:1-6. 